\section{Suicide, the main cause of referral of patients from a general hospital for psychiatric evaluation}

\author{
Elena Gómez ${ }^{1}$, Marianela Guzmán² and Oriol Yuguero Torres ${ }^{1,2 *}$ \\ ${ }^{1}$ Faculty of Medicine, University of Lleida, Lleida, Spain \\ ${ }^{2}$ Biomedical Research Institute of Lleida (IRBLLEIDA, Lleida, Spain
}

\section{Abstract}

Introduction: Psychiatric emergencies constitute between 1\% - 10\% of general emergencies in Spain, of which a quarter will end up being hospital admissions. There is little literature on patients who are referred from general to specialized hospitals.

Methods: Cross-Sectional study of all the patients referred in 2018. Sociodemographic variables and clinical variables, such as diagnosis and discharge destination, were analyzed. An analysis was performed by comparing means with the Chi Square test.

Results: 433 derived emergencies were analyzed. Most of the patients were women. The main reason for derivation were suicide attempts. Of those patients, $40 \%$ required hospitalizations. More than $50 \%$ of the derived emergencies were referred to the home and followed up on an outpatient basis.

Conclusion: Most derived emergencies are referred to the home after evaluation. This implies that most are not life-threatening and that with adequate tools for evaluating suicide risk, we could avoid transfers and improve the resolution of these cases.

\section{More Information}

*Address for Correspondence: Oriol Yuguero Torres, Biomedical Research Institute of Lleida, Avda Rovira Roure 80, 25198 Lleida, Tel: + 34973705200 ;

Email: Oriol.yuguero@gmail.com

Submitted: March 20, 2021

Approved: April 06, 2021

Published: April 07, 2021

How to cite this article: Gómez E, Guzmán M, Torres OY. Suicide, the main cause of referral of patients from a general hospital for psychiatric evaluation. Arch Psychiatr Ment Health. 2021; 5: 018-021.

DOI: 10.29328/journal.apmh.1001030

Copyright: @ 2021 Gómez E, et al. This is an open access article distributed under the Creative Commons Attribution License, which permits unrestricted use, distribution, and reproduction in any medium, provided the original work is properly cited.

Keywords: Emergencies; Suicide; Management

\section{(D) Check for updates}

OPen Access

\section{Introduction}

Psychiatric emergencies constitute between $1-10 \%$ of general emergencies in Spain [1], of which a quarter will end up being hospital admissions. Many organic pathologies can manifest psychiatric symptoms, and vice versa. Because of this it is very important to have general knowledge in both areas, since the reasons for urgent psychiatric consultation are often very imprecise. Multiple studies show the most frequent reasons of urgency at the state level, which are: decompensations of organic pathology, confusional states, acute delusions/hallucinations disorders, mood disorders (depression, euphoria, mania), as well as conduct disorder and suicide attempt.

The definition of psychiatric emergency [2] is imprecise, since the life risk remains being present, and this appears in relation to a disturbance in thought, in affect or behavior, requiring immediate therapeutic intervention. In this type of situations, urgent attention will take into account the special nature of the psychiatric pathology as well as the set of organic pathologies.
Over the years, international classifications, different psychotherapies and psychopharmacology have been made. This represents a big change in the approach to mental illnesses, since emergency services began to differentiate according to the pathology presented. Currently, urgent psychiatric pathology care can be attended in the emergency services of hospitals general, as well as in exclusive mental health centers [3]. In our territory, Psychiatric Emergencies are attended in a specialized Hospital, although patients also go to the General Hospital of the same city.

The purpose of this study is to identify the characteristics of those emergencies that, after being assessed in an Emergency Service, require a specialized assessment by the Hospital's on-call Psychiatry team of Lleida`s University Hospital Santa María.

\section{Material and methods}

Retrospective cross sectional study of patients treated with urgent psychiatric pathology in Lleida`s Hospital Arnau de Vilanova (HUAV) that are referred for evaluation at Hospital Santa María during 2018. The HUAV is the reference hospital 
in the city with a population of more than 300,000 people The Santa María University Hospital is a public center specialized in Mental Health and geriatric care.

\section{Inclusion criteria}

All the patients attended and refereed to the Psychiatry team were included.

\section{Variables}

Dependent variable: Diagnosis at the Emergency Episode.

Independent variables: Referral after care in the Psychiatry Service: home discharge, hospital admission, transfer to other centers, voluntary discharge, and escape or abandonment of the center; called "Destination".

Other sociodemographic variables were included just like age and gender.

\section{Statistical analysis}

A descriptive analysis of the sample was carried out, later we proceeded to establish the relationships of the independent variables and each set of pathologies by a bivariate analysis with graphic representation of the results. The co-relationship between means was performed using the Chi Square test. The statistical significance was defined as $p<0.05$.

\section{Ethical considerations}

The study was approved by the Ethics Research Committee of the Biomedical Research Institute of Lleida.

\section{Results}

In this study we included all the patients referred from the general Hospital for psychiatric evaluation. 433 patients derived from the Emergency Service to the Specialized Hospital were evaluated. Of this group, 152 were men (35\%) and $64.8 \%$ were women. The first cause of referral was suicide attempts (29.1\%). As we can see in table 1, the second cause were organic psychiatric disorders and the third one were problems due to drug abuse $(19,2 \%)$. The rest of the pathologies are described in table 1 . Table 2 shows the final destination of the referrals, $54 \%$ of the patients are discharged at home after the assessment. However, close to $40 \%$ of the patients need hospital admission after psychiatric evaluation. Two patients leave voluntary the hospital prior to end the medical evaluation. Table 3 shows the derivation in function of the diagnosis. There is a significant association between diagnosis and discharge destination, especially in patients attended for suicidal ideas.

In the study we have seen that the diagnosis is statistically associated with the destination of the patient. Patients with suicide attempts are the most frequently referred to their home and admitted, with percentages of $30.1 \%$ and $31.4 \%$ respectively. In second place, the pathologies that

\begin{tabular}{|c|c|c|}
\hline Table 1: Patients' diagnosis. & N & \\
\hline Diagnose & 129 & $29.8 \%$ \\
\hline Suicide attempt & 83 & $19.2 \%$ \\
\hline Drug abuse & 29 & $6.7 \%$ \\
\hline Depression & 23 & $5.3 \%$ \\
\hline Anxiety & 29 & $6.7 \%$ \\
\hline Personality disorders & 25 & $5.8 \%$ \\
\hline Schizophrenia & 5 & $1.2 \%$ \\
\hline Cognitive impairment & 87 & $20.1 \%$ \\
\hline Other organ-based disorders & 23 & $5.3 \%$ \\
\hline Delirium & 433 & $100 \%$ \\
\hline Total & & \\
\hline
\end{tabular}

Table 2: Destination upon discharge after evaluation in a Specialized Hospital.

\begin{tabular}{|c|c|c|}
\hline Destination & N & Percentage \\
\hline Home & 237 & $54.7 \%$ \\
\hline Hospital Admission & 172 & $39.7 \%$ \\
\hline Voluntary Discharge & 2 & $0.5 \%$ \\
\hline Referral to other centers & 14 & $3.2 \%$ \\
\hline Leaving hospital & 8 & $1.8 \%$ \\
\hline Total & 433 & $100 \%$ \\
\hline
\end{tabular}

are most often referred at home and admitted are organbased disorders, with $21.1 \%$ and 19,2\%. These percentages represent a wide variety of pathologies that may debut or have any presentation of a psychiatric nature.

Third, drug use is the most relevant group of pathology, with a $20.7 \%$ of patients referred to their home and $15.1 \%$ who remain admitted.

Alcohol consumption stands out, either as acute intoxication or in patients with chronic alcoholism followed by cannabis use.

A less frequent situation was the patients who abandoned the emergency department, including those who used toxic substances, followed by suicide attempts, which represented $1.8 \%$ of the total.

Finally, the lowest presentation was voluntary discharge, with a percentage of $0.5 \%$ of the total, which was equally relevant in patients who presented drug use as well as personality disorders.

No statistical association was found between gender, age and diagnosis.

\section{Discussion}

Most of the emergencies that are referred to specialized psychiatric services are sent home. Many patients with acute behavioral or mental health emergencies use the emergency department for their care [4].

Suicidal ideas are the main reason of referral for specialized evaluation, as other studies described previously [5].

In 2019, 2265 psychiatric pathology emergencies were treated at the Arnau de Vilanova Hospital emergency department, and 433 of these, were referred to Santa María 
Table 3: Destination upon discharge depending on patients' diagnosis.

\begin{tabular}{|c|c|c|c|c|c|c|c|}
\hline & \multicolumn{7}{|c|}{ Destination } \\
\hline Diagnosis & & Home & Hospital Admission & Voluntary discharge & Referral to other centers & Leaving Hospital & Total \\
\hline \multirow{3}{*}{ Suicide attempt } & $\mathrm{N}$ & 71 & 54 & 0 & 2 & 2 & 129 \\
\hline & \% inside "Diagnosis" & $55.0 \%$ & $41.9 \%$ & $0.0 \%$ & $1.6 \%$ & $1.6 \%$ & $100 \%$ \\
\hline & \% inside "Destination" & $30.0 \%$ & $31.4 \%$ & $0.0 \%$ & $14.3 \%$ & $25.0 \%$ & $29.8 \%$ \\
\hline \multirow{3}{*}{ Drug abuse } & $\mathrm{N}$ & 49 & 26 & 1 & 4 & 3 & 83 \\
\hline & \% inside "Diagnosis" & $59.0 \%$ & $31.3 \%$ & $1.2 \%$ & $4.8 \%$ & $3.6 \%$ & $100 \%$ \\
\hline & $\%$ inside "Destination" & $20.7 \%$ & $15.1 \%$ & $50.0 \%$ & $28.6 \%$ & $37.5 \%$ & $19.2 \%$ \\
\hline \multirow{3}{*}{ Depression } & $\mathrm{N}$ & 10 & 19 & 0 & 0 & 0 & 29 \\
\hline & \% inside "Diagnosis" & $34.5 \%$ & $65.5 \%$ & $0.0 \%$ & $0.0 \%$ & $0.0 \%$ & $100 \%$ \\
\hline & $\%$ inside "Destination" & $4.2 \%$ & $11.0 \%$ & $0.0 \%$ & $0.0 \%$ & $0.0 \%$ & $6.7 \%$ \\
\hline \multirow{3}{*}{ Anxiety } & $\mathrm{N}$ & 22 & 1 & 0 & 0 & 0 & 23 \\
\hline & \% inside "Diagnosis" & $95.7 \%$ & $4.3 \%$ & $0.0 \%$ & $0.0 \%$ & $0.0 \%$ & $100 \%$ \\
\hline & $\%$ inside "Destination" & $9.3 \%$ & $0.6 \%$ & $0.0 \%$ & $0.0 \%$ & $0.0 \%$ & $5.3 \%$ \\
\hline \multirow{3}{*}{ Personality disorders } & $\mathrm{N}$ & 21 & 6 & 1 & 0 & 1 & 29 \\
\hline & \% inside "Diagnosis" & $72.4 \%$ & $20.7 \%$ & $3.4 \%$ & $0.0 \%$ & $3.4 \%$ & $100 \%$ \\
\hline & $\%$ inside "Destination" & $8.9 \%$ & $3.5 \%$ & $50.0 \%$ & $0.0 \%$ & $12.5 \%$ & $6.7 \%$ \\
\hline \multirow{3}{*}{ Schizophrenia } & $\mathrm{N}$ & 4 & 17 & 0 & 4 & 0 & 25 \\
\hline & \% inside "Diagnosis" & $16.0 \%$ & $68.0 \%$ & $0.0 \%$ & $16.0 \%$ & $0.0 \%$ & $100 \%$ \\
\hline & \% inside "Destination" & $1.7 \%$ & $9.9 \%$ & $0.0 \%$ & $28.6 \%$ & $0.0 \%$ & $5.8 \%$ \\
\hline \multirow{3}{*}{ Cognitive impairment } & $\mathrm{N}$ & 2 & 3 & 0 & 0 & 0 & 5 \\
\hline & \% inside "Diagnosis" & $40.0 \%$ & $60.0 \%$ & $0.0 \%$ & $0.0 \%$ & $0.0 \%$ & $100 \%$ \\
\hline & \% inside "Destination" & $0.8 \%$ & $1.7 \%$ & $0.0 \%$ & $0.0 \%$ & $0.0 \%$ & $1.2 \%$ \\
\hline \multirow{3}{*}{ Other organ-based disorders } & $\mathrm{N}$ & 50 & 34 & 0 & 2 & 1 & 87 \\
\hline & \% inside "Diagnosis" & $57.5 \%$ & $39.1 \%$ & $0.0 \%$ & $2.3 \%$ & $1.1 \%$ & $100 \%$ \\
\hline & \% inside "Destination" & $21.1 \%$ & $19.8 \%$ & $0.0 \%$ & $14.3 \%$ & $12.5 \%$ & $20.1 \%$ \\
\hline \multirow{3}{*}{ Delirium } & $\mathrm{N}$ & 8 & 12 & 0 & 2 & 1 & 23 \\
\hline & \% inside "Diagnosis" & $34.8 \%$ & $52.2 \%$ & $0.0 \%$ & $8.7 \%$ & $4.3 \%$ & $100 \%$ \\
\hline & $\%$ inside "Destination" & $3.4 \%$ & $7.0 \%$ & $0.0 \%$ & $14.3 \%$ & $12.5 \%$ & $5.3 \%$ \\
\hline \multirow{3}{*}{ Total } & $\mathrm{N}$ & 237 & 172 & 2 & 14 & 8 & 433 \\
\hline & $\%$ inside "Diagnosis" & $54.7 \%$ & $39.7 \%$ & $0.5 \%$ & $3.2 \%$ & $1.8 \%$ & $100 \%$ \\
\hline & \% inside "Destination" & $100 \%$ & $100 \%$ & $100 \%$ & $100 \%$ & $100 \%$ & $100 \%$ \\
\hline
\end{tabular}

Hospital, which represents $19.11 \%$ of the total. This means that about $80 \%$ of psychiatric pathology that visits the emergency department is solved by the Emergency Service (ES) professionals, and the patients who were derived, most were sent home, which shows the importance of carrying out a correct ambulatory follow-up, from primary care professionals and mental health centers [6].

This fact reveals that, despite being an emergency situation, after an evaluation by the medical team, it is actually a reversible situation, without the need for hospital admission and continuous surveillance. Nowadays the Emergency Services are over-saturated with patients who do not require urgent priority attention [7].

Secondly, 38\% of the referred patients were admitted, being in this group most frequently suicide attempts, as well as underlying organic disorders.

The organic psychiatric disorders are frequently treated in the emergency department usually for hospital admission, because they are usually exacerbations of chronic diseases, or atypical presentations, but always with an organic base.

$1.8 \%$ of the patients requested voluntary discharge. In these cases it would be interesting know the degree of competence of these patients, due to the challenge represented by evaluating patients with psychiatric disease.
The most derived pathology, which is the suicide attempts, has been the subject of multiple studies [8], and it is interesting that practically half of the patients are discharged home. The assessment of suicide risk [9] is really important to know it those patients can back home or need more assistance.

This fact is related to the establishment of the "Codi risc de suïcidi" [10] by the Department of Health. It is a protocol of action in cases of suicide attempt, which through various scales (such as the "Sad Persons Scale"), will classify patients according to risk in their attempt. In relation to our study, if the patient is at low risk, the indication for follow-up is at home or outpatient, while if the risk is high, hospital admission will be considered. But assessing this suicidal risk is something that generally requires specialists in Mental Health, and that is why they are the most derived group.

Regarding acute drug poisoning, they go to the emergency room for atypical consumption of substances that patients consume regularly. These patients are not admitted, because it is not a first episode of intoxication, and once the effect has passed, the symptoms pf the patients are usually reversible.

\section{Conclusion}

The main cause of referral from a general hospital for psychiatric assistance is the attention to people who has tried to suicide. Close to $50 \%$ of those patients require 
hospitalization. This is really important, because shows the importance of psychiatric evaluation and the importance of team work between general and specialist emergency teams.

In our opinion, the key in the care and referral of patients with mental health problems is the presentation of the process, whether is acute or chronic, the frequency of attention and knowing the limitations of general attention. It is important to make society aware that the emergency departments in the field of psychiatry are available to care for patients needing hospitalization or specialized attention. However, team work between different hospitals has shown that is useful for giving the best attention to the patients.

\section{Highlights}

- Suicide attempts are the most common cause of referral to a specialized hospital.

- After psychiatric evaluation, majority of patients are discharged to home.

- $1,8 \%$ of those patients leave the hospital without a competence evaluation.

\section{References}

1. García FM, Boada SS, Collsamata AX, Joaquim IG, Pérez YA, et al. Meteorological factors and psychiatric emergencies. Actas Espanolas de Psiquiatria. 2009; 37: 34-41.

PubMed: https://pubmed.ncbi.nlm.nih.gov/18803032/

2. Wheat S, Dschida D, Talen MR. Psychiatric Emergencies. Primary Care. 2016; 43: 341-354.

PubMed: https://pubmed.ncbi.nlm.nih.gov/27262012/
3. Lozano M, O’Neill A, Jorda L, Vázquez A. The psychiatric emergency in the general hospital: an analysis of the indications for hospitalization. Actas Luso Esp Neurol Psiquiatr Cienc Afines. 1992; 20: 208-213. PubMed: https://pubmed.ncbi.nlm.nih.gov/1462805/

4. Nentwich LM, Wittmann CW. Emergency Department Evaluation of the Adult Psychiatric Patient. Emergency Medicine Clinics of North America. 2020; 38: 419-435.

PubMed: https://pubmed.ncbi.nIm.nih.gov/32336334/

5. RoelandsM,DeschepperR,BilsenJ.Psychiatric ConsultationandReferral of Persons Who Have Attempted Suicide. Crisis. 2017; 38: 261-268. PubMed: https://pubmed.ncbi.nlm.nih.gov/28228063/

6. Hernández-Viadel $M$, Cañete Nicolás $C$, Pérez Prieto JF, Lera Calatayud G, Gómez Beneyto M. Evaluation of the efficacy of involuntary outpatient treatment in reducing the use of mental health services in hospital. Rev Psiquiatr Salud Ment. 2010; 3: 50-54. PubMed: https://pubmed.ncbi.nlm.nih.gov/23445929/

7. González-Peredo R, Prieto-Salceda MD, Campos-Caubet L, Fernández-Díaz F, García-Lago-Sierra Á, et al. Characteristics of hospital emergency departments users. Frequent Users. 2018; 44: 537-548.

8. Goñi-Sarriés A, Janda-Galán L, Macaya-Aranguren $P$, Azcárate $L$, López-Goñi JJ, et al. Differences between suicide attempts and other psychiatric hospital emergencies. Actas Espanolas de Psiquiatria. 2018; 46: 83-91.

9. Weber AN, Michail M, Thompson A, Fiedorowicz JG. Psychiatric Emergencies: Assessing and Managing Suicidal Ideation. The Medical Clinics of North America. 2017; 101: 553-571.

PubMed: https://pubmed.ncbi.nlm.nih.gov/28372713/

10. Mortier P, Vilagut G, Puértolas Gracia B, De Inés Trujillo A, Alayo Bueno I, et al. Catalonia Suicide Risk Code Epidemiology (CSRC-Epi) study: protocol for a population-representative nested case-control study of suicide attempts in Catalonia. Spain. BMJ Open. 2020; 10: e037365. PubMed: https://pubmed.ncbi.nlm.nih.gov/32660952/ 